\title{
Fístula ureterocutánea tras nefrectomía: aportación de dos casos y revisión de la literatura
}

\author{
Vázquez Alonso F, Vicente Prados FJ, Pascual Geler M, Funes Padilla C, Cózar Olmo JM, \\ Tallada Buñuel M.
}

Servicio de Urología. Hospital Universitario Virgen de las Nieves. Granada.

Actas Urol Esp 2008;32(9):931-933

\begin{abstract}
RESUMEN
FÍSTULA URETEROCUTÁNEA TRAS NEFRECTOMÍA: APORTACIÓN DE DOS CASOS Y REVISIÓN DE LA LITERATURA

Las fístulas ureterales se relacionan con la cirugía ginecológica, cirugía digestiva y cirugía urológica reconstructiva del aparato urinario superior. Están descritas fístulas ureterovaginales, ureteroduodenales, ureterocolónicas, ureteropleurales, ureterovasculares, etc.

Sin embargo, las fístulas ureterocutáneas del muñón ureteral tras nefrectomía son una entidad muy inusual. Presentamos dos casos así como su resolución mediante cirugía.

Palabras clave: Fístula uro-cutáneas. Complicaciones quirúrgicas. Muñón ureteral. Nefrectomía.

\section{ABSTRACT}

URETEROCUTANEOUS FISTULA AFTER NEPHRECTOMY: TWO CASES

The ureteral fistulas are related to the gynecological surgery, digestive surgery and reconstructive urologic surgery of the upper urinary tract. Fistulas are described ureterovaginal, ureteroduodenal, ureterocolonic, ureteropleural, ureterovascular, etc.

However, the ureterocutaneous fistulas of the ureteral stump after nephrectomy are a very unusual entity. We report two cases as well as their resolution by means of surgery.

Keywords: Uro-cutaneous fistula. Surgical complications. Ureteral stump. Nephrectomy.
\end{abstract}

$\mathrm{L}$ a aparición de una fístula ureterocutánea tras nefrectomía es una complicación poco referida en la literatura, que sin embargo hay que tener en cuenta especialmente tras cirugías complejas. Presentamos dos casos clínicos así como su resolución quirúrgica.

\section{CASOS CLÍNICOS}

\section{Caso 1}

Mujer de 53 años de edad con antecedentes personales de obesidad mórbida y colecistectomía por colelitiasis. Presenta una historia de litiasis renal tratada con litotricia extracorpórea en múltiples ocasiones, que evoluciona a una litiasis coraliforme de riñón derecho con pérdida de función del mismo. Tras varios episodios de pielonefritis decidimos rea- lizar nefrectomía derecha por lumbotomía (Anatomía Patológica: Atrofia renal con reacción inflamatoria inespecífica).

En el postoperatorio inmediato presenta dolor abdominal difuso e intenso secundario a peritonitis aguda. Tras laparotomía exploradora se objetiva perforación colónica derecha que obliga a realizar una hemicolectomía con anastomosis termino-terminal.

La evolución es tórpida con infección de la herida quirúrgica que precisa Friedrich y resutura.

A los 2 meses de la cirugía la enferma refiere emisión de material purulento a través de un orificio próximo a la cicatriz de lumbotomía que persiste durante 10 meses a pesar de las curas locales. Tras realizar fistulograma a través del orificio apre- 
ciamos una comunicación entre un trayecto fistuloso a nivel de la pared y el muñón ureteral no pasando el contraste a vejiga (Fig. 1).

Tras descartar mediante cistografía miccional la ausencia de reflujo que pudiera ser el causante de la fístula, proponemos tratamiento quirúrgico, que consiste en realizar una fistulorrafia con escisión del trayecto fistuloso a nivel de la pared y ureterectomía. A nivel del cabo proximal de uréter apreciamos una importante reacción a cuerpo extraño (seda). Por último ligamos el extremo ureteral con material reabsorbible.

La evolución postoperatoria fue favorable, estando en la actualidad la paciente asintomática.

\section{Caso 2}

Varón de 72 años, con antecedentes personales de adenomectomía transvesical. Es diagnosticado de atrofia renal derecha secundaria a estenosis de la unión pieloureteral, con frecuentes episodios de pie-

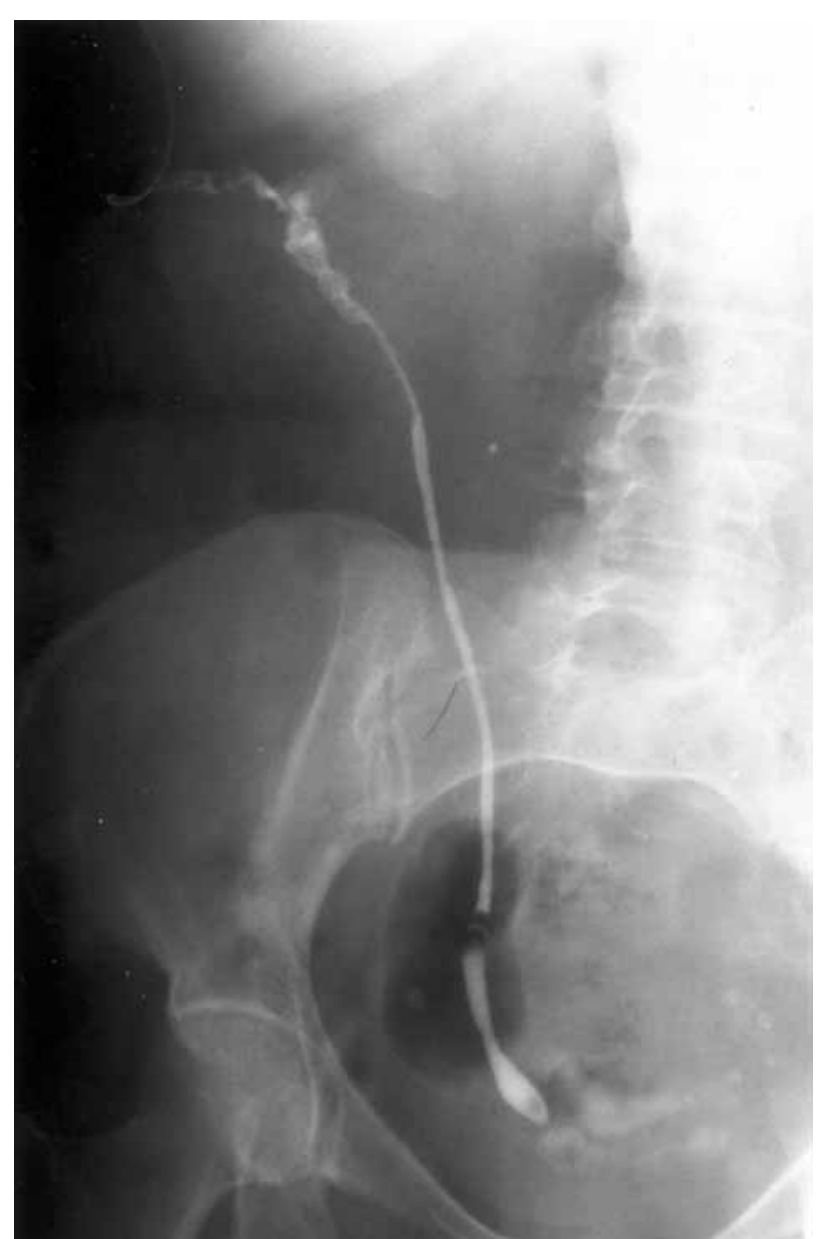

FIGURA 1. Fístula ureterocutánea (visión anteroposterior). lonefritis. Se interviene practicándose nefrectomía subcapsular derecha mediante lumbotomía subcostal. La cirugía fue dificultosa por la existencia de gran componente fibroso y pielonefrítico. La herida quirúrgica evoluciona de forma tórpida persistiendo la salida de material purulento a través de la propia incisión durante más de dos meses, por lo que realizamos una fistulografía objetivando un trayecto fistuloso desde piel hasta el muñón ureteral derecho (Fig 2).

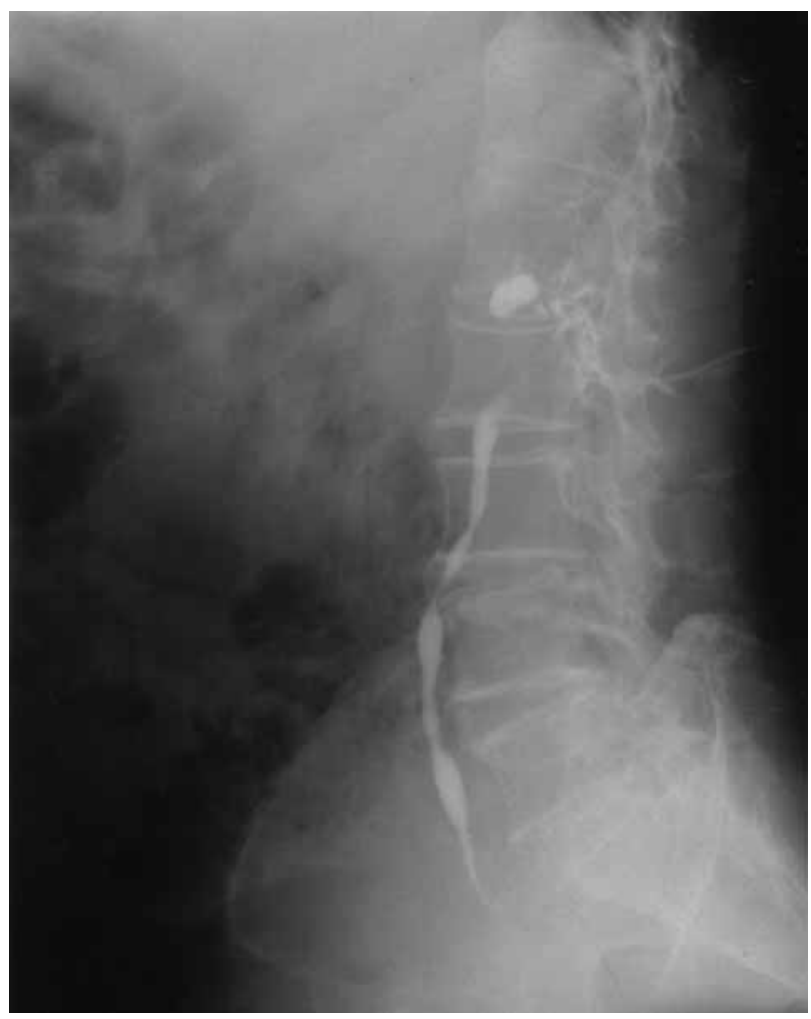

FIGURA 2. Fistula ureterocutánea (visión lateral).

Proponemos tratamiento quirúrgico procediendo a la escisión del trayecto fistuloso y ureterectomía, con evolución postquirúrgica satisfactoria.

Actualmente el paciente se encuentra asintomático, con herida bien cicatrizada y controles analíticos, ecográficos y urografía intravenosa normales.

\section{DISCUSIÓN}

Las fístulas urocutáneas se definen como la comunicación anómala entre la vía urinaria y la piel, y representan una entidad poco frecuente. Las fístulas ureterales, aunque pueden aparecer de modo espontáneo son más frecuentes como complicación de la cirugía ginecológica, digestiva y urológica reconstructiva del aparato urinario superior. 
Dentro de las distintas variedades de fístulas ureterales (ureterovaginales, ureteroduodenales, ureterocolónicas, ureterovasculares, etc.), las fístulas ureterocutáneas representan una modalidad muy poco frecuente ${ }^{1}$.

El desarrollo de una fístula ureterocutánea tras la realización de una nefrectomía es un hallazgo excepcional con muy pocos casos descritos en la literatura, apareciendo tras la cirugía de procesos renales inflamatorios crónicos y pielonefritis xantogranulomatosas ${ }^{2,3}$.

La presentación clínica más común es la aparición de débito de material líquido a través de la herida al poco tiempo de la cirugía, aunque se pueden presentar al cabo de muchos años ${ }^{4}$.

El diagnóstico de una fístula ureterocutánea tras una nefrectomía se debe llevar a cabo mediante la realización de una fistulografía que manifestará la comunicación existente entre la piel y el muñón ureteral. También sería recomendable descartar la existencia de un reflujo vesicoureteral como factor predisponente de la fístula.

En cuanto al tratamiento, la escisión quirúrgica es una opción válida en fístulas tras nefrectomía, representando el tratamiento gold-standard ${ }^{5}$. En los casos en los que se asocie a reflujo vesicoureteral, una opción interesante sería la inyección endoscópica subtrigonal de politetrafluoroetileno (Teflón) ${ }^{6}$.

\section{CONCLUSIÓN}

Las fístulas ureterocutáneas tras nefrectomía son una entidad poco frecuente que hay que sospechar tras cirugías complejas en las que se objetive débito de material líquido a través de la herida. El diagnóstico se debe confirmar mediante una fistulografía, descartando la existencia de reflujo vesicoureteral. La escisión quirúrgica de la fístula es una opción válida.

\section{REFERENCIAS}

1. Allona Almagro A, Sanz Migueláñez JL, Pérez Sanz P, Pozo Mengual B, Navío Niño S. Fístulas urinarias: puesta al día. Actas Urol Esp. 2002;26(10):776-795.

2. Belis JA, McCuskey BM. Ureterocutaneous fistula following nephrectomy. W V Med J. 1980;76(3):49-50.

3. Warnock N, O'Flynn KJ, Thomas DG. Xanthogranulomatous pyelonephritis and ureterocutaneous fistula. Br J Urol. 1991;67 (5):549-550.

4. Shahidi S, Fries J, Kay L. An ureterocutaneous fistula forty years after nephrectomy. Scand J Urol Nephrol. 2000;34(4):282-283.

5. Sharma SK, Perry KT, Turk TM. Endoscopic injection of fibrin glue for the treatment of urinary-tract pathology. J Endourol. 2005; 19(3):419-423.

6. Saslawsky M, Niederberger C, Schacht M, Prinz L, Ross L. Ureterocutaneous fistula: a case report of treatment by subtrigonal injection of polytetrafluoroethylene (Sting procedure). J Urol. 1989;142(5): 1310-1311.

Correspondencia autor: Dr. F. Vázquez Alonso

Servicio de Urología. Hospital Universitario Virgen de las Nieves Avda. de las Fuerzas Armadas, 2 - 18014 Granada

Tel.: 958020145

E-mail: fernando.vazquez.alonso.sspa@juntadeandalucia.es Información artículo: Nota clínica

Trabajo recibido: febrero 2007

Trabajo aceptado: marzo 2007 